Structural Eng. / Earthquake Eng. Vol.5. No.1, 61s-69s. April 1988

Japan Society of Civil Engineers (Proc. of JSCE No.392/I-9)

\title{
DYNAMIC STABILITY OF AN INITIALLY DEFLECTED RECTANGULAR PLATE UNDER AN INPLANE DYNAMIC MOMENT
}

\author{
By Kazuo TAKAHASHI*, Yasunori KONISHI**, Ryuta KAWANO*** \\ and Shigeo URAKAWA****
}

\begin{abstract}
In the following paper, the vibration of an initially deflected rectangular plate under a sinusoidally time-varying inplane moment is examined from the point of view of dynamic instability. The equation of motion describing the large deflection of the initially deflected plate is analyzed by the Galerkin method. The resulting equations for time variables are integrated by using the Runge-Kutta-Gill method. The dynamic instability regions are analyzed by the small deflection theory of a thin plate, neglecting nonlinear terms. The amplitudes of unstable regions are determined by large deflection theory.

Numerical results are presented for various shapes and magnitudes of the initial deflection. The effect of the initial deflection on natural frequency, dynamic instability and amplitudes are discussed.

Keywords: dynamic stability, nonlinear vibration, vibration of plate
\end{abstract}

\section{INTRODUCTION}

Out-of-plane vibrations of a rectangular plate under a sinusiodally time-varying inplane moment are known to be caused by dynamic instability. Unstable regions and their amplitudes in out-of-plane vibration induced by in-plane moment are still not clear analytically. The preceding study by the authors, reference (1), clarified the existence of unstable regions in the small deflection theory, their mechanisms and the basic properties of the dynamic instability problem. They are : (1) Unstable regions of combination resonances are predominant for the present case. This fact is different from unstable regions of a plate with a uniformly distributed load which has simple parametric resonances only. The unstable regions of the combination resonance are wide when natural frequencies of the rectangular plate related to unstable motions are close to each other, independently of the boundary conditions and aspect ratios; (2) The static moment influences the unstable regions. The widths of simple parametric resonances which are narrow in the absence of the static moment, grow wide.

Next, the amplitudes of unstable motions were estimated by the large deflection theory of a plate ${ }^{2) .31}$. The conclusions are as follows : (3) The amplitudes of the out-of-plane vibrations of the rectangular plate subjected to the inplane dynamic moment are bounded due to the geometric nonlinear terms effect. The amplitudes of combination resonances are greater than those of simple parametric resonances for the present case; (4) The effect of the static moment generally increases the amplitude of the unstable

* Member of JSCE, Dr. Eng. , Associate Professor, Department of Civil Egnineering, Nagasaki University (Bunkyo-machi 1-14. Nagasaki City)

** Member of JSCE, Dr. Eng., Professor, Department of Civil Engineering, Nagasaki University (Bunkyo-machi 1-14, Nagasaki City)

*** Member of JSCE, Graduate Student of Nagasaki University (Bunkyo-machi 1-14, Nagasaki City)

**** Member of JSCE, Chuo Consultant Co., Ltd. (Arato 1-1-6. Fukuoka City, Fukuoka) 
motions. The amplitudes of the combination resonances whose modal shapes are similar to those of the buckling mode are greatly affected by the static moment.

These conclusions were obtained for plate with no initial deflection. However, it is a well-known phenomenon that the initial deflection of the plate induces additional out-of-plane deflection under the action of an inplane moment ${ }^{4}$. The influence of the initial deflections on the out-of-plane deflection has already been discussed in connection with the initiation of fatigue cracks by Maeda and Okurat). The response of the plate subjected to the repeated moment is essentially a vibration problem. Therefore, it is necessary to consider the dynamic instability of the initially deflected plate.

Based upon this point, Kuranishi et al. ${ }^{5)}$ studied the dynamic response of an initially deflected web plate under periodic beam bending. However, the relation between initial deflection and dynamic stability is still not clear.

This paper shows an analytical approach to the investigation of the dynamic stability of the initially deflected rectangular plate subjected to an inplane dynamic moment. The equation of motion describing large deflection of the initially deflected plate is analyzed by the Galerkin method. The resulting coupled nonlinear Mathieu equations for time variables are integrated by using the Runge-Kutta-Gill method. Numerical results are shown for various shapes and magnitudes of the initial deflection. Natural frequencies and unstable regions are obtained by neglecting nonlinear terms and maximum amplitudes are obtained.

\section{GOVERNING EQUATIONS AND BOUNDARY CONDITIONS}

A cartesian co-ordinate $\operatorname{system}(x, y)$ is introduced as shown in Fig. 1. Static moment $M_{0}$ and sinusoidally time-varying moment $M_{t} \cos \Omega t$ act on two edges, $x=0$ and $a$ and an inplane force $N_{x}$ due to these moments is given by

$$
N_{x}=\frac{6}{b^{2}}\left(1-2 \frac{y}{b}\right)\left(M_{0}+M_{t} \cos \Omega t\right)
$$

where $b$ is the length of the loaded edge, and $M_{t}$ and $\Omega$ are the amplitudes of the sinusoidally time-varying moment and the forcing circular frequency respectively.

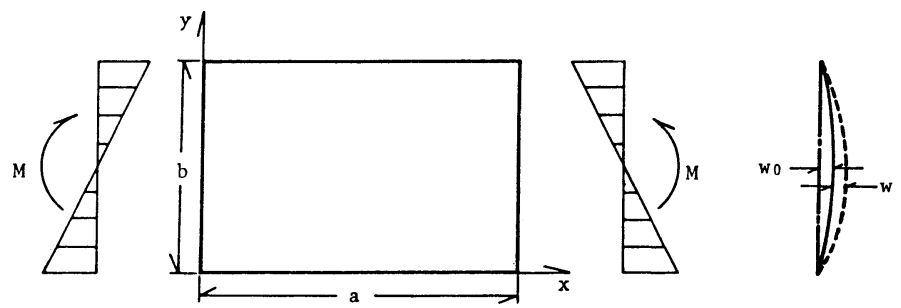

Fig. 1 Geometry and coordinate system.

Assuming that the effects of the longitudinal and rotational inertia forces and transverse shear can be neglected, then the basic equations for large amplitude free vibrations of a rectangular plate with initial deflection $w_{0}$ subjected to an inplane moment can be written using the Marguerre equation as

$$
\begin{aligned}
& L(w, F)= \rho d \frac{\partial^{2} w}{\partial t^{2}}+D \nabla^{4} w-\frac{6}{b^{2}}\left(1-2 \frac{y}{b}\right)\left(M_{0}+M_{t} \cos \Omega t\right) \frac{\partial^{2} w}{\partial x^{2}} \\
&-d\left\{\frac{\partial^{2} F}{\partial y^{2}} \cdot \frac{\partial^{2}\left(w+w_{0}\right)}{\partial x^{2}}+\frac{\partial^{2} F}{\partial x^{2}} \cdot \frac{\partial^{2}\left(w+w_{0}\right)}{\partial y^{2}}-2 \frac{\partial^{2} F}{\partial x \partial y} \cdot \frac{\partial^{2}\left(w+w_{0}\right)}{\partial x \partial y}\right\}=0 \\
& \nabla^{4} F=E\left[\left\{\frac{\partial^{2}\left(w+w_{0}\right)}{\partial x \partial y}\right\}^{2}-\frac{\partial^{2}\left(w+w_{0}\right)}{\partial x^{2}} \cdot \frac{\partial^{2}\left(w+w_{0}\right)}{\partial y^{2}}-\left\{\left(\frac{\partial^{2} w_{0}}{\partial x \partial y}\right)^{2}-\frac{\partial^{2} w_{0}}{\partial x^{2}} \cdot \frac{\partial^{2} w_{0}}{\partial y^{2}}\right\}\right) \cdots
\end{aligned}
$$


were $w$ denotes the plate deflection, $\rho$ the mass density, $d$ the plate thickness, $t$ the time, $D=E d^{3} /$ $\left\{12\left(1-v^{2}\right)\right\}$ is the bending stiffness, $E$ Young's modulus, $v$ Poisson's ratio and $F$ Airy's stress function.

The following boundary conditions for bending and inplane displacement are considered in the present analysis :

Simply supported along all edges : i.e.,

$$
w=\frac{\partial^{2} w}{\partial x^{2}}=0 \quad(x=0, a), \quad w=\frac{\partial^{2} w}{\partial y^{2}}=0 \quad(y=0, b)
$$

All edges immovable in the inplane directions:

$$
\int_{0}^{b} u d y=0 \quad(x=0, a), \quad \int_{0}^{a} v d x=0 \quad(y=0, b)
$$

where

$$
\begin{aligned}
& u=\int_{0}^{x} \frac{\partial u}{\partial x} d x=\int_{0}^{x}\left[\frac{1}{E}\left(\frac{\partial^{2} F}{\partial y^{2}}-\nu \frac{\partial^{2} F}{\partial x^{2}}\right)-\frac{1}{2}\left\{\frac{\partial\left(w+w_{0}\right)}{\partial x}\right\}^{2}+\frac{1}{2}\left(\frac{\partial w}{\partial x}\right)^{2}\right] d x \\
& v=\int_{0}^{y} \frac{\partial v}{\partial y} d y=\int_{0}^{y}\left[\frac{1}{E}\left(\frac{\partial^{2} F}{\partial x^{2}}-\nu \frac{\partial^{2} F}{\partial y^{2}}\right)-\frac{1}{2}\left\{\frac{\partial\left(w+w_{0}\right)}{\partial y}\right\}^{2}+\frac{1}{2}\left(\frac{\partial w}{\partial y}\right)^{2}\right] d y
\end{aligned}
$$

in which $u$ and $v$ are inplane displacements in the $x$ and $y$ directions, respectively.

\section{METHOD OF SOLUTION}

Taking these boundary conditions into account, we assume the solution of equation (2) by

$$
w=d \sum_{n=1} T_{M n}(t) \cdot W_{M n}(x, y)
$$

where $T_{M n}(t)$ is an unknown function of the time variable, and $W_{M n}(x, y)$ an eigen-function associated with free vibrations satisfying the geometric boundary conditions of the plate, defined as

$$
W_{M n}=\sin \frac{M \pi x}{a} \sin \frac{n \pi y}{b}
$$

in which $M$ and $n$ are half-wave numbers in the $x$ and $y$ directions, respectively. We assume the initial deflection to satisfy the boundary conditions as follows:

$$
w_{0}=e_{0} d W_{G H}(x, y)
$$

where $e_{0}$ is the nondimensional amplitude of the initial deflection and $W_{G H}$ the shape function; $G$ and $H$ are half-wave numbers in the $x$ and $y$ directions, respectively.

The general solution of stress function $F$ of equation (3) may be expressed in the form :

$F=F_{p}+F_{c}$

where $F_{\rho}$ is the particular solution and $F_{c}$ the complementary solution. By substituting equations $(7)$ and (8) into equation (3), the particular solution $F_{p}$ can be obtained as shown in Appendix A. Let the complementary solution $F_{c}$ be expressed as in references 4$\left.), 5\right), 6$ ) as

$$
F_{c}=A(t) x^{2}+B(t) y^{2}
$$

where $A(t)$ and $B(t)$ are functions of time, determined so as to satisfy the inplane boundary condition (4-b) (Appendix B).

After substituting equations (5), (7) and ( 8 ) into equation (2), we apply the Galerkin method to obtain

$$
\begin{aligned}
& \frac{\rho d b^{4}}{D} I_{M p}^{1} \ddot{T}_{M p}+I_{M p}^{2} T_{M p}+\frac{6}{D}\left(M_{0}+M_{t} \cos \Omega t\right) \sum_{n=1} I_{M \rho n} T_{M n}+\sum_{n=1} \sum_{r=1} \sum_{s=1} I_{M n r s p} T_{M n} T_{M r} T_{M S} \\
& +e_{0} \sum_{n=1} \sum_{r=1} I_{M n \text { M } \mathrm{P}} T_{M n} T_{M r}+e_{0}^{2} \sum_{n=1} I_{M n C H \rho} T_{M n}=0
\end{aligned}
$$

where $I_{M n}^{1}, I_{M n}^{2}, I_{M n p}$ and $I_{M n r s p}$ are integrations that appear in the process of the Galerkin method (Appendix C) and $p=1,2, \cdots$.

Using nondimensional notations, we obtain the following differential equation for the time variable : 


$$
\begin{aligned}
& \ddot{T}_{M p}+\left(\frac{\omega_{p}^{M}}{\omega_{1}^{1}}\right)^{2} T_{M p}+\left(\bar{M}_{0}+\bar{M}_{t} \cos \bar{\omega} \tau\right) \sum_{n=1} A_{M p n} T_{M n}+\sum_{n=1} \sum_{r=1} \sum_{s=1} B_{M n r s p} T_{M n} T_{M r} T_{M s} \\
& +e_{0} \sum_{n=1} \sum_{r=1} C_{M n r G H \rho} T_{M n} T_{M r}+e_{0}^{2} \sum_{n=1} D_{M n G H \rho} T_{M n}=0
\end{aligned}
$$

where $\bar{M}_{0}=M_{0} / M_{c r}$ is the nondimensional static moment, $\bar{M}_{t}=M_{t} / M_{c r}$ the nondimensional dynamic moment, $M_{c r}=\lambda_{c r} \pi^{2} D / 6$ the buckling moment, $\lambda_{c r}$ the eigen-value of the buckling moment $\bar{\omega}=\Omega / \Omega_{1}^{1}$ the nondimensional forcing circular frequency, $\Omega_{1}^{1}=\alpha_{1}^{1} \pi^{2} / b^{2} \sqrt{D / \rho d}$ the first natural circular frequency of the unloaded plate, $\alpha_{1}^{1}$ the first eigen-value of vibration, $\tau=\Omega_{1}^{1} t$ the non-dimensional time, and $\omega_{p}^{M}$ the $p$-th natural circular frequency with the half-wave number $M . A_{M n p}$ and $B_{M n r s p}$ being given in Appendix D. As can be seen in equation (11), the second order of the intial deflection $\left(e_{0}^{2}\right)$ is included in the linear terms. The first order of the initial deflection $\left(e_{0}\right)$ is included in the nonlinear terms. It is assumed that the initial deflection is much more important in the nonlinear case. The parameters in equation (11) are the modes $(G, H)$ (half-wave numbers, $G$ and $H)$ and magnitude $\left(e_{0}\right)$ of the initial deflection, static moment $\bar{M}_{0}$, amplitude $\bar{M}_{t}$ of the dynamic moment and the forcing circular frequency $\bar{\omega}$. The stability of the rectangular plate with the particular value of $G, H, e_{0}$ and $\bar{M}_{0}$ will have unstable solutions for some combinations of $\bar{M}_{t}$ and $\bar{\omega}$.

\section{METHOD OF TIME RESPONSE ANALYSIS}

Time variable $T_{M p}$ of equation (11) will be integrated numerically by using the Runge-Kutta-Gill method. From the results of the preceding papers ${ }^{1) .2)}$, a summing type combination resonance near frequency $\bar{\omega}=$ $\left(\omega_{i}^{M}+\omega_{j}^{M}\right) / k(k=1,2, \cdots)$ is predominant and the simple parametric resonance near frequency $\bar{\omega}=2 \omega_{i}^{M} / k$ through coupling terms is not important in the present case (see page 181 of reference 1 ). The notation $\omega_{i}^{M}$ is a nodimensional natural frequency of the plate defined as $\Omega_{i}^{M} / \Omega_{1}^{1}$ where $\Omega_{i}^{M}$ is the natural frequency with the mode $(M, i)$ of the initially deflected plate, $M$ is the half-wave number in the $x$ direction and $i$ or $j$ is the half-wave number in the $y$ direction. The combination resonance is a coupling of vibrations with two different modes, $(M, i)$ and $(M, j)$. The two degree-of-freedom approach by using two modes which induce unstable motions is adopted to obtain a time response analysis. The purpose of the present analysis is to obtain the unstable regions which occur under the assumptions of the small deflection theory and to determine the amplitude of the unstable motions. Therefore, the initial conditions for the time variable are set equal to $T_{M i}=T_{M j}=0.01$ and $\dot{T}_{M i}=\dot{T}_{M j}=0.0$ to satisfy the small amplitude vibration. Poisson's ratio $\nu$ of the plate is assumed to be 0.3 and the effect of the static moment $\bar{M}_{0}$ is not considered here.

\section{NATURAL FREQUENCY}

Unstable motions occur near forcing frequency $\bar{\omega}$ such as $2 \omega_{i}^{M} / k$ and $\left(\omega_{i}^{M}+\omega_{j}^{M}\right) / k$. Therefore, it is necessary to clarify the effect of the initial deflection on the natural frequency of the rectangular plate. By neglecting nonlinear terms in equation (11) and putting $\bar{M}_{t}=0.0$ and $\bar{M}_{0}=0.0$, the free vibrations of the initially deflected plate can be obtained. By using this equation, the natural frequencies of the initially deflected plate are obtained for the mode $(G, H)$ of the initial deflection and its magnitude $e_{0}$.

The natural frequencies of the square plate with half-wave numbers $M$ equal to 1 and 2 in
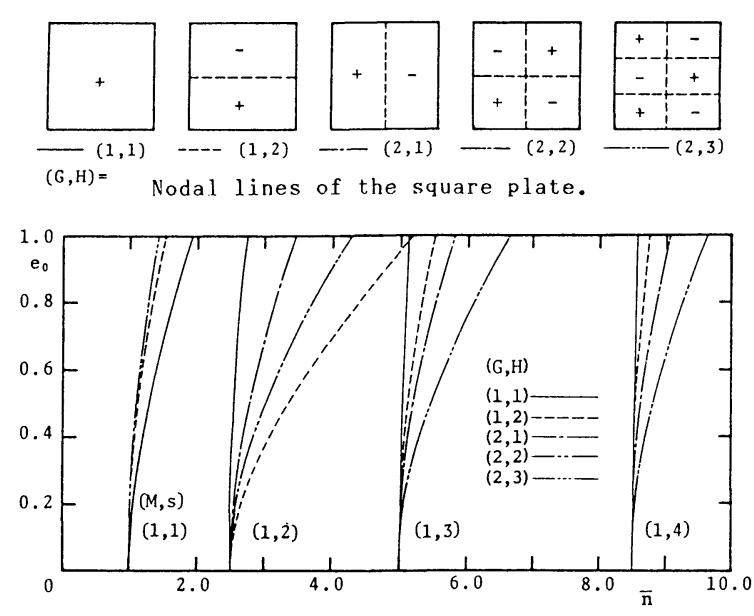

Fig. 2 Magnitude $e_{0}$ of the intial deflection vs natural frequency $\bar{n}$ for the case of $M=1$. 
the $x$ direction are illustreted for various modes $(G, H)$ of the initial deflection in Figs. 2 and 3. In these figures, the ordinate $e_{0}$ shows the magnitude of the initial deflection normalized for the plate thickness, while the abscissa $\bar{n}=\Omega_{s}^{M} / \Omega_{1}^{1}$ stands for the natural frequency of the initially deflected plate normalized for the lowest natural frequency. The corresponding mode of vibration is shown by notation $(M, s)$ for various modes $(G, H)$ of the initial imperfection. In general, natural

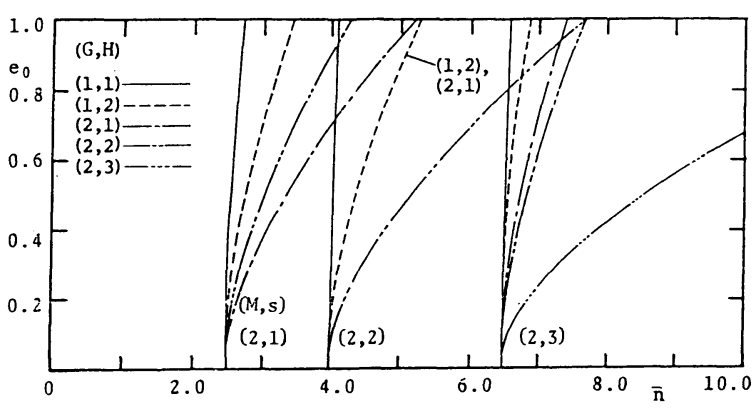

Fig. 3 Magnitude $e_{0}$ of the intial deflection vs natural frequency $\bar{n}$ for the case of $M=2$. frequencies grow larger with increase of the magnitude of the initial deflection. The amount of increase is significant for cases in which the numbers $(M, s)$ of half-waves of the mode of vibration coincide with those of the modes $(G, H)$ of the initial deflection.

\section{UNSTABLE REGIONS}

The effect of the shape $(G, H)$ and its maginitude $e_{0}$ of initial deflection on the unstable regions where the out-of-plane vibrations of the plate occur, is clarified in this section. From the results of the dynamic stability analysis ${ }^{11}$, it is concluded that combination resonances are predominant for plates subjected to inplane moment and simple parametric resonance is negligible. In this case, only combination resonances are considered.

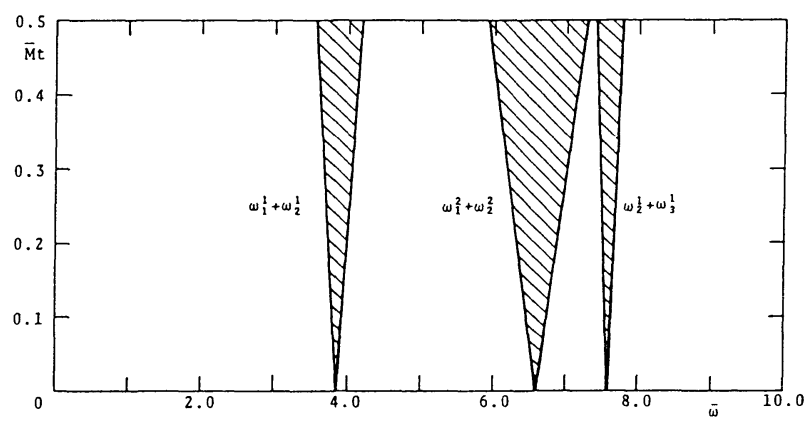

Fig. 4 Unstable regions of a square plate $: e_{0}=0.5, G=1$ and $H=1$.

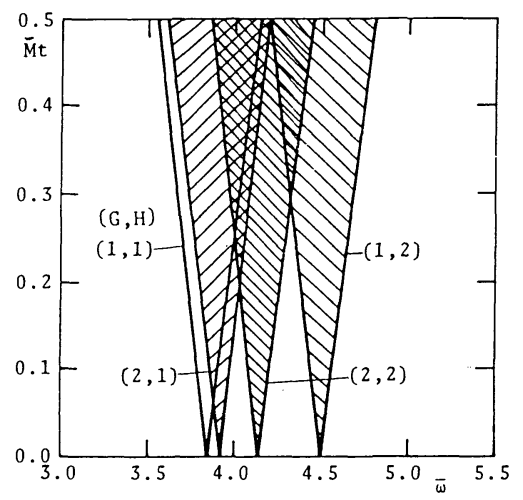

Fig. 5 Unstable regions of a square plate : $\omega_{1}^{1}+\omega_{2}^{1}$ and $e_{0}=0.5$.

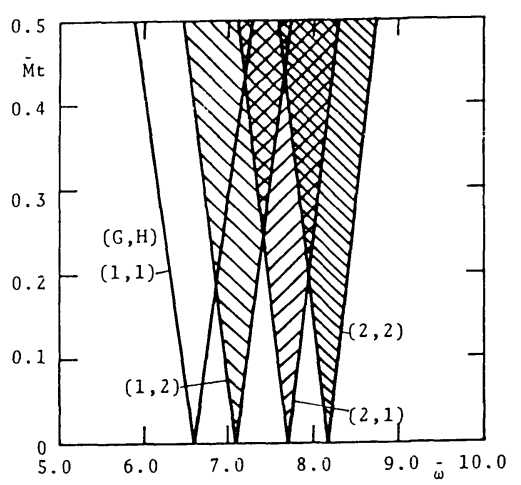

Fig. 6 Unstable regions of a square plate $: \omega_{1}^{2}+\omega_{2}^{2}$ and $e_{0}=0.5$. 
Fig. 4 shows unstable regions for the initially deflected square plate with shape $(G, H)=(1,1), e_{0}=$ 0.5 and $\bar{M}_{0}=0.0$. In this figure, the ordinate $\bar{M}_{t}=M_{t} / M_{c r}$ denotes the amplitude of the periodic force normalized for the corresponding buckling momemt, while the abscissa $\bar{\omega}=\Omega / \Omega_{1}^{1}$ stands for the exiting frequency normalized for the lowest natural frequency. Furthermore, the hatched portions represent the regions of instability where the out-of-plane vibrations of the plate are excited. The notation $\omega_{1}^{M}+\omega_{j}^{M}$ shows the unstable motion of the combination resonance with two modal shapes $(M, i)$ and $(M, j)$.

(1) Effect of the shape of the initial deflection

Figs. 5 and 6 show the width of the unstable motion $\omega_{1}^{1}+\omega_{2}^{1}$ (for $M=1$ ) and $\omega_{1}^{2}+\omega_{2}^{2}$ (for $M=2$ ) of the square plate with $e_{0}=0.5$ for various shapes $(G, H)$ of the initial deflection. As the natural frequency $\omega_{i}^{M}$ changes depending on the shape $(G, H)$ of the initial deflection, the frequency where the unstable region occurs moves to the higher frequency range. Therefore, the effect of the initial deflection $(G, H)$ is different for $M=1$ and $M=2$, as can be seen in Figs. 5 and 6 .

Table 1 shows the upper and lower boundaries of the unstable motion of $\omega_{1}^{2}+\omega_{2}^{2}$ for $\bar{M}_{t}=0.5$. The unstable region becomes narrower with increases in the frequency range where the unstable motions occurs.

(2) Effect of the magnitude of the initial deflection

Fig. 7 shows the widths of the unstable motion $\omega_{1}^{1}+\omega_{2}^{1}$ (for $M=1$ ) of the square plate with $(G, H)=$ $(2,2)$ for various magnitudes $e_{0}$ of the initial deflection. As natural frequency $\omega_{i}^{M}$ increases with increases in the magnitude $e_{0}$ of the initial deflection, the frequency range where the unstable region occurs moves to the higher frequency range. Table 2 shows the upper and lower boundaries of the unstable motion $\omega_{1}^{2}+\omega_{2}^{2}$ for $\bar{M}_{t}=0.5$. The unstable region becomes narrower with increases in the magnitude $e_{0}$ of the initial deflection. The widths of the unstable region are narrow in the presence of the initial deflection.

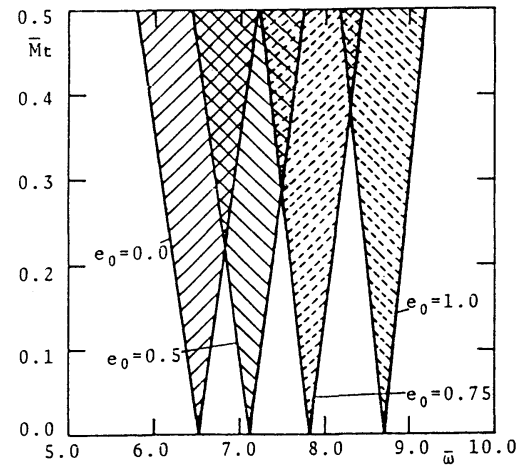

Fig. 7 Unstable regions of a square plate: $\omega_{1}^{2}+\omega_{2}^{2}$. $G=2$ and $H=2$.
Table 1 Upper-lower boundaries of the unstable region : $\omega_{1}^{2}+\omega_{2}^{2}, \bar{M}_{t}=0.5$ and $e_{0}=0.5$.

\begin{tabular}{l|rc|l}
\hline$(\mathrm{G}, H)$ & \multicolumn{1}{|c|}{$\omega_{\text {10wer }} \omega_{\text {upper }}$} & width \\
\hline$(1,1)$ & 5.90 & $\sim 7.25$ & 1.35 \\
$(1,2)$ & $6.45-7.75$ & 1.30 \\
$(2,1)$ & $7.15 \sim 8.30$ & 1.15 \\
$(2,2)$ & $7.60 \sim 8.75$ & 1.15 \\
\hline
\end{tabular}

Table 2 Upper-lower boundaries of the unstable region : $\omega_{1}^{2}+\omega_{2}^{2}, \bar{M}_{t}=0.5, \quad G=2$ and $H$ $=2$.

\begin{tabular}{c|cc|c}
\hline$e_{0}$ & $\omega_{\text {1ower }} \omega$ upper & width \\
\hline 0.00 & $5.80-7.20$ & 1.40 \\
0.25 & $5.95-7.35$ & 1.40 \\
0.50 & $6.45-7.75$ & 1.30 \\
0.75 & $7.25-8.40$ & 1.15 \\
1.00 & $8.20-9.20$ & 1.00 \\
\hline
\end{tabular}

\section{MAXIMUM AMPLITUDES}

The nonlinear response of the unstable motion can be obtained by using numerical integrations of equation (11). The nonlinear time response for the combination resonance $\omega_{1}^{2}+\omega_{2}^{2}$ is shown in Fig. 8 . The quadratic nonlinear terms are included in the equation of motion in conjunction with the initial deflection. As the quadratic nonlinear terms have non-symmetric restoring force properties, the maximum amplitudes with positive and negative signs are not the same. This result shows that the maximum deflection becomes large when the plate experiences initial deflection. 


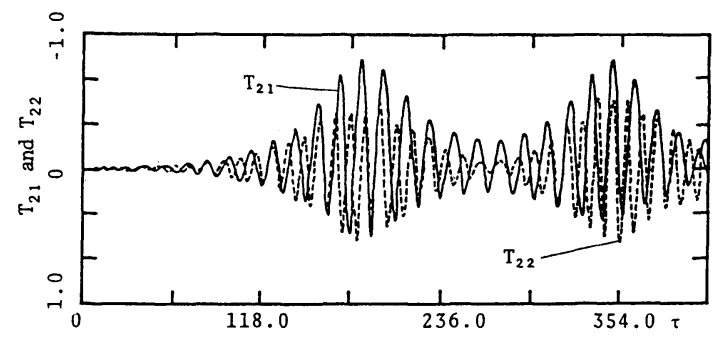

Fig. 8 Time history of the combination resonance : $\omega_{1}^{2}+\omega_{2}^{2}, \quad e_{0}=0.4, \quad G=2, H=1, \bar{M}_{t}=0.3$ and $\bar{\omega}=7.35$.

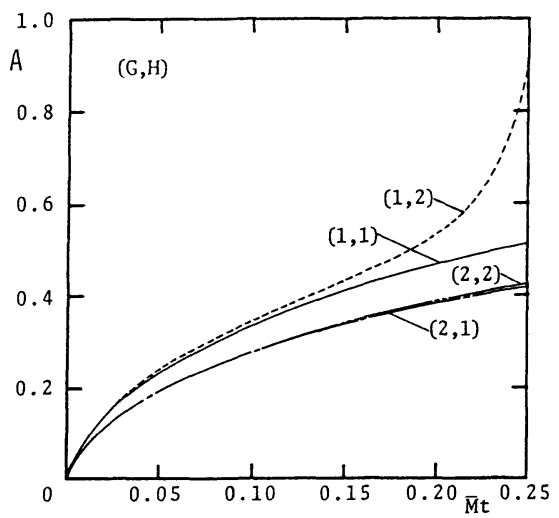

Fig. 9 Maximum amplitudes of unstable motions : $\omega_{1}^{1}+\omega_{2}^{1}$ and $e_{0}=0.2$.

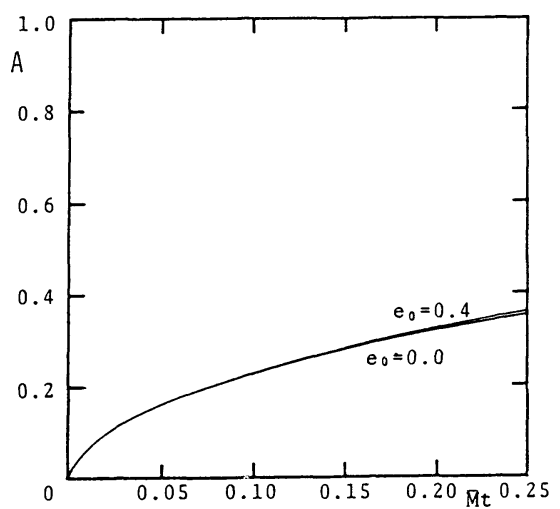

Fig. 11 Maximum amplitudes of unstable motions : $\omega_{1}^{2}+\omega_{2}^{2}$, $G=1$ and $H=2$.

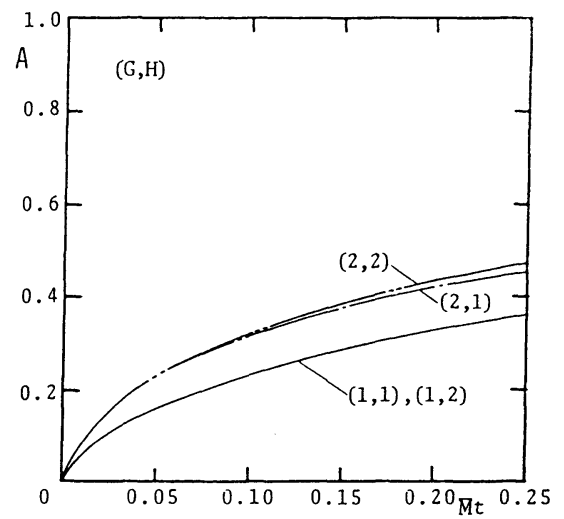

Fig. 10 Maximum amplitudes of unstable motions: $\omega_{1}^{2}+\omega_{2}^{2}$ and $e_{0}=0.2$.

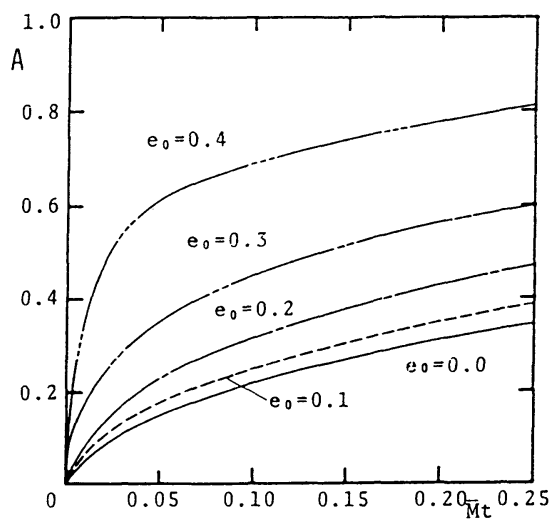

Fig. 12 Maximum amplitudes of unstable motions: $\omega_{1}^{2}+\omega_{2}^{2}$, $G=2$ and $H=2$.

\section{(1) Effect of the mode of initial deflection}

Figs. 9 and 10 show the amplitudes of the unstable motions $\omega_{1}^{1}+\omega_{2}^{1}$ (for $\left.M=1\right)$ and $\omega_{1}^{2}+\omega_{2}^{2}$ (for $\left.M=2\right)$ of the square plate with $e_{0}=0.2$ for various shapes $(G, H)$ of the initial deflection. In these figures, the abscissa $\bar{M}_{t}$ shows the nondimensional moment and the ordinate $\mathrm{A}$ indicates the maximum amplitude which is nondimensionalized by the plate thickness $d$. The maximum amplitudes are affected by the shape $(G, H)$ of the initial deflection. The amplitudes of unstable motion become large when the half-wave number $M$ in the $x$ direction coincides with that of the initial deflection $G$ as in $M=1$ and $G=1$ in Fig. 9 and $M=2$ and $G=2$ in Fig. 10. 


\section{(2) Effect of the magnitude of initial deflection}

Figs. 11 and 12 show the maximum amplitude $A$ of the unstable motion $\omega_{1}^{2}+\omega_{2}^{2}$ of a square plate with the shapes $(G, H)=(1,2)$ and $(2,2)$ of the initial deflection. The amplitudes of the initially deflected plate with $(G, H)=(1,2)$ almost coincide with those of plates with no initial deflection as in Fig. 11. That is, the amplitude of the unstable motion is not affected by the initial deflection when the half-wave number $M$ is not equal to that of the initial deflection $G$. On the other hand, when $M$ is equal to $G$, the maximum amplitude is greatly affected by the shape $(G, H)$ of the initial deflection as shown in Fig. 12. From these results, it is clear that the maximum amplitude is dependent on the combination of the shape of the initial deflection and the mode of vibration. When $M$ is equal to $G$, the effect of the second order of nonlinear terms becomes significant. The nonlinear time response in this case is similar to that of Fig. 8 . The difference between plus and minus amplitudes becomes large and the maximum amplitude $A$ increases with the increase of the magnitude $e_{0}$. On the other hand, when $M$ is not equal to $G$, the effect of the quadratic nonlinear terms is not significant. The time response in the present case is similar to that of a plate with no initial deflection as shown in Fig. 4 in reference 3.

\section{CONCLUSIONS}

The present paper shows the dynamic instability of a rectangular plate with an initial deflection subjected to a dynamic inplane moment. The dynamic instability is clarified for various shapes and amplitudes of the initial deflection. The main results obtained may be summarized as follows:

(1) The natural frequency of a rectangular plate with initial deflection increases with the increase of the amplitude of the initial deflection. Its magnitude depends on the modal shape of the initial deflection and is the largest when the wave number of the mode of vibration in the $x$ direction coincides with that of the initial deflection.

(2) The frequency where unstable motions occur changes depending on the shape and magnitude of the initial deflection. The frequency moves to the higher frequency range with increases in the amplitude of the initial deflection and its width becomes narrow in the presence of the initial deflection.

(3) The quadratic nonlinear terms attributed to the initial deflection are included in the equation of motion. The quadratic nonlinear terms modify the property of restoring forces and the plus and minus amplitudes are not the same. The maximum amplitudes increase when this term is predominant.

(4) The effect of the initial deflection on the maximum response is conspicuous when its shape along the loaded direction coincides with the mode of vibration. The maximum amplitudes increase with increase of the amplitude of the initial deflection.

\section{APPENDIX A : Particular Solution $\boldsymbol{F}_{p}$ of Equation ( 8 )}

where

$$
F_{\rho}=E d^{2} \sum_{n=1} \sum_{s=1} T_{M n} T_{M S} F_{M n s}^{p}+e_{0}^{2} E d^{2} \sum_{n=1} T_{M n} E_{M n C H}^{p}
$$

$$
\begin{aligned}
F_{M n s}^{p}= & A_{n s}^{M} \cos (n-s) \pi \eta+B_{n s}^{M} \cos (n+s) \pi \eta+\cos 2 M \pi \xi\left\{C_{n s}^{M} \cos (n-s) \pi \eta+D_{n s}^{M} \cos (n+s) \pi \eta\right\}, \\
E_{M n G H}^{D}= & \cos (M-G) \pi \xi\left\{E_{n H}^{M G} \cos (n-H) \pi \eta+F_{n H}^{M G} \cos (n+H) \pi \eta\right\} \\
& +\cos (M+G) \pi \xi\left\{G_{n H}^{M G} \cos (n-H) \pi \eta+H_{n H}^{M G} \cos (n+H) \pi \eta\right\}, \\
A_{n s}^{M}= & \frac{M^{2} s}{4 \mu^{2}(n-s)^{3}}, \quad B_{n s}^{M}=\frac{M^{2} s}{4 \mu^{2}(n+s)^{3}}, \quad C_{n s}^{M}=\frac{\mu^{2} M^{2}(n+s) s}{4\left\{4 M^{2}+(n-s)^{2} \mu^{2}\right\}^{2}}, \quad D_{n s}^{M}=\frac{\mu^{2} M^{2}(n-s) s}{4\left\{4 M^{2}+(n+s)^{2} \mu^{2}\right\}^{2}}, \\
E_{n H}^{M G}= & \frac{-\mu^{2}(M H-n G)^{2}}{4\left\{(M-G)^{2}+(n-H)^{2} \mu^{2}\right\}^{2}}, \quad F_{n H}^{M G}=\frac{\mu^{2}(M H+n G)^{2}}{4\left\{(M-G)^{2}+(n+H)^{2} \mu^{2}\right\}^{2}}, \\
G_{n H}^{M G}= & \frac{\mu^{2}(M H+n G)^{2}}{4\left\{(M+G)^{2}+(n-H)^{2} \mu^{2}\right\}^{2}}, \quad H_{n H}^{M G}=\frac{-\mu^{2}(M H-n G)^{2}}{4\left\{(M+G)^{2}+(n+H)^{2} \mu^{2}\right\}^{2}}
\end{aligned}
$$

where

$A_{n s}^{M}=0 \quad(n=s), \quad \xi=x / a, \quad \eta=y / b, \quad \mu=a / b \quad$ (aspect ratio) 


\section{APPENDIX B : Particular Solution $\boldsymbol{F}_{p}$ of Equation (9)}

$$
F_{c}=E d^{2} \sum_{n=1} \sum_{s=1} T_{M n} T_{M S} F_{M n s}^{c}+e_{0} E d^{2} \sum_{n=1} T_{M n} E_{M n G H}^{c}
$$

where

$$
\begin{aligned}
& F_{M n s}^{c}=\frac{\pi^{2} \delta_{n s}}{16\left(1-\nu^{2}\right)}\left\{\left(\mu^{2} n s+\nu M^{2}\right) \xi^{2}+\left(M^{2} / \mu^{2}+\nu n s\right) \eta^{2}\right\}, \\
& E_{M n G H}^{c}=\frac{\pi^{2} \delta_{M G} \delta_{n H}}{8\left(1-\nu^{2}\right)}\left\{\left(\mu^{2} H n+\nu M G\right) \xi^{2}+\left(M G / \mu^{2}+\nu H n\right) \eta^{2}\right\}
\end{aligned}
$$

\section{APPENDIX C : Integrations of the Galerkin Method}

$$
\begin{aligned}
& I_{M p}^{1}=\int_{0}^{1} \int_{0}^{1} W_{M p}^{2} d \xi d \eta \\
& I_{M p}^{2}=\int_{0}^{1} \int_{0}^{1}\left(\frac{1}{\mu^{4}} \frac{\partial^{4} W_{M p}}{\partial \xi^{4}}+\frac{2}{\mu^{2}} \frac{\partial^{4} W_{M p}}{\partial \xi^{2} \partial \eta^{2}}+\frac{\partial^{4} W_{M p}}{\partial \eta^{4}}\right) W_{M p} d \xi d \eta, \\
& I_{M n p}=-\frac{1}{\mu^{2}} \int_{0}^{1} \int_{0}^{1}(1-2 \eta) \frac{\partial^{2} W_{M n}}{\partial \xi^{2}} W_{M p} d \xi d \eta \\
& I_{M n r s p}=-\frac{12\left(1-\nu^{2}\right)}{\mu^{2}} \int_{0}^{1} \int_{0}^{1}\left(\frac{\partial^{2} F_{M r s}}{\partial \eta^{2}} \frac{\partial^{2} W_{M n}}{\partial \xi^{2}}-2 \frac{\partial^{2} F_{M r s}}{\partial \xi \partial \eta} \frac{\partial^{2} W_{M n}}{\partial \xi \partial \eta}+\frac{\partial^{2} F_{M r s}}{\partial \xi^{2}} \frac{\partial^{2} W_{M n}}{\partial \eta^{2}}\right) W_{M p} d \xi d \eta, \\
& \left.+\frac{\partial^{2} E_{M r G H}}{\partial \eta^{2}} \frac{\partial^{2} W_{M n}}{\partial \xi^{2}}-2 \frac{\partial^{2} E_{M r G H}}{\partial \xi \partial \eta} \frac{\partial^{2} W_{M n}}{\partial \xi \partial \eta}+\frac{\partial^{2} E_{M r G H}}{\partial \xi^{2}} \frac{\partial^{2} W_{M n}}{\partial \eta^{2}}\right) W_{M p} d \xi d \eta, \\
& F_{M r s}=F_{M r S}^{p}+F_{M r s}^{c}, \quad E_{M r G H}=E_{M r G H}^{p}+E_{M r G H}^{c} \\
& A_{M n \rho}=I_{M n \rho} /\left\{I_{M p}^{1}\left(\alpha_{1}^{1}\right)^{2} \pi^{4}\right\}, \quad B_{M n r s p}=\lambda_{c r} I_{M n r s \rho} /\left\{I_{M p}^{1}\left(\alpha_{1}^{1}\right)^{2} \pi^{2}\right\}, \\
& C_{\text {MnrGH }}=I_{\text {MnrGH }} /\left\{I_{M p}^{1}\left(\alpha_{1}^{1}\right)^{2} \pi^{4}\right\}, \quad D_{M r G H p}=I_{M r G H p} /\left\{I_{M p}^{1}\left(\alpha_{1}^{1}\right)^{2} \pi^{4}\right\} \\
& \Omega_{p}^{M}=\sqrt{\frac{I_{M p}^{2}}{I_{M p}^{1}} \frac{D}{\rho d b^{4}}}
\end{aligned}
$$

\section{APPENDIX D : Coefficients of Equation (11)}$$
I_{M n r G H P}=-\frac{12\left(1-\nu^{2}\right)}{\mu^{2}} \int_{0}^{1} \int_{0}^{1}\left(\frac{\partial^{2} F_{M r s}}{\partial \eta^{2}} \frac{\partial^{2} W_{G H}}{\partial \xi^{2}}-2 \frac{\partial^{2} F_{M r s}}{\partial \xi \partial \eta} \frac{\partial^{2} W_{G H}}{\partial \xi \partial \eta}+\frac{\partial^{2} F_{M r s}}{\partial \xi^{2}} \frac{\partial^{2} W_{G H}}{\partial \eta^{2}}\right.
$$$$
I_{M r G H \rho}=-\frac{12\left(1-\nu^{2}\right)}{\mu^{2}} \int_{0}^{1} \int_{0}^{1}\left(\frac{\partial^{2} E_{M r G H}}{\partial \eta^{2}} \frac{\partial^{2} W_{G H}}{\partial \xi^{2}}-2 \frac{\partial^{2} E_{M r G H}}{\partial \xi \partial \eta} \frac{\partial^{2} W_{G H}}{\partial \xi \partial \eta}+\frac{\partial^{2} E_{M r G H}}{\partial \xi^{2}} \frac{\partial^{2} W_{G H}}{\partial \eta^{2}}\right) W_{M p} d \xi d \eta
$$

where

\section{REFERENCES}

1) Takahashi, K., Tagawa, M., Ikeda, T. and Matsukawa, T. : Dynamic Stability of a Rectangular Plate Subjected to Inplane Moment, Proc. of JSCE, No. 341, pp. 179 186, 1984.

2) Takahashi, K., Ikeda, T. and Kawano, R. : Nonlinear Response of a Rectangular Plate Subjected to Inplane Moment, Journal of Structural Engineering, Vol. 32A, pp. 705 714, 1986.

3) Takahashi, K. and Konishi, Y. and Kawano, R. : Nonlinear Response of a Rectangular Plate Subjected to Inplane Dynamic Moment, Proc. of JSCE, No. 374/I-6, pp. 358 369, 1986.

4) Maeda, Y. and Okura, I. : Effects of Initial Deflection of Fatigue Cracks due to Out-of-Plane Deflection of Thin Plate, Proc. of JSCE, No. 329, pp. 1 11, 1982

5) Kuranishi, S., Fukaya, S. and Shima, T. : Vibration of an Initially Deflected Web Plate under Periodic Beam Bending, Proc. of JSCE, No. 341, pp. 229 232, 1984.

6) Marguerre, K. : Zur Theorie der gekrummten Platte grosser Formanderung, Proc. of 5th International Congress for Applied Mechanics, pp.93〜101, 1938.

7) Timoshenko, S. P. and Woinowsky-Krieger, S. : Theory of Plates and Shells, 2nd Edition, McGraw-Hill Book Co., pp. 425 428, 1955 